PAOLA COPPI

Università di Verona, Italia

Recibido: 21/07/2015

jrccop@tin.it

Aprobado: 27/02/2016

\title{
María Zambrano interpreta Spinoza e Hegel
}

\section{María Zambrano interprets Spinoza and Hegel}

RESUMEN: María Zambrano empieza su estudio de Spinoza con su tesi doctoral La salvacíon del individuo en Spinoza. El filósofo neerlandés es, para ella, una persona clave y fundamental dentro de la evolución de la filosofía, sobre todo por el concepto que Spinoza tiene de la Totalidad, de Dios como causa sui y Deus sive natura. La especulación filosófica de la filósofa andaluza finaliza, sin embargo, con Hegel, de quien interpreta, de una manera original, la idea de la Historia como experiencia implacable y necesaria del Geist.

PALABRAS CLAVE: SALVACÍON - INDIVIDUO - TOTALIDAD - DEUS - NATURALEZA.
ABSTRACT: María Zambrano begins her study of Spinoza with her doctoral thesis La salvacíon del individuo en Spinoza. The Dutch philosopher is, for her, a milestone in the evolution of philosophy, especially for Spinoza's concept of Totality, of Deus causa sui and Deus sive natura. The philosophical speculation of the Spanish philosopher ends, however, with Hegel, whose idea of history as a necessary and relentless experience of the Geist, she interpretes in an original way.

KEY WORDS: SALVATION - INDIVIDUAL - TOTALITY - GOD - NATURE 
$\mathrm{D}$ AL 1931 AL 1936 María Zambrano viene nominata «profesora ayudante», in sostituzione di Xavier Zubiri, titolare di metafisica all’Università di Madrid.

Zambrano ha già pubblicato il suo primo saggio Horizonte del liberalismo (1930) e numerosi articoli per diverse riviste, tra le più importanti, la Revista de Occidente e El Liberal. Allo stesso tempo sta per terminare la sua tesi dottorale dal titolo, La salvacíon del individuo en Spinoza. Un lavoro, in verità, mai concluso che, se da un lato mostra tutta l'influenza ricevuta dal suo maestro, il filosofo Ortega y Gasset, profondo conoscitore di Spinoza ${ }^{1}$; dall'altro, invece, sottolinea la personale rielaborazione del pensiero spinoziano e, in particolare, dell'Etica che Zambrano definisce il diamante di pura luce.

La filosofa andalusa studia Spinoza con passione rimanendo folgorata, in particolare, dal concetto di totalità: ovvero dalla idea che Dio è causa sui, e pertanto è causa di sé e di tutto, e della natura e dell'uomo. Come già si è detto, Zambrano non concluderà mai la sua tesi. Tuttavia è possibile ricostruire in parte il suo contenuto grazie a due saggi conservati oggi nell'A rchivio della Fundación María Zambrano in Vélez-Málaga. Il primo é il manoscritto A.F.M.Z

[1] Zambrano frequenta le lezioni di Ortega. E in particolare quelle del corso «Il sistema di Spinoza e il sistema di Fichte» (1930-1931). Nella nota preparatoria al corso Ortega si domanda il perché si debba scegliere come oggetto di un corso di filosofia un autore come Spinoza. E la risposta è la seguente: 1) in primo luogo perché Spinoza è la figura del passato filosofico meno conosciuta e studiata; 2) in secondo luogo, perché Spinoza è così poco conosciuto e studiato che solo ora, attraverso il recupero del suo studio finalmente è possibile presentare una nuova sua interpretazione, più acuta e fertile di quelle giunte fino ad ora; 3 ) inoltre Spinoza è l'anello fondamentale di una gloriosa catena dellevoluzione filosofica del XVII secolo; 4) infine perché Spinoza pone al centro della riflessione filosofica il grande problema dell'essere dell'uomo. Ciò che Zambrano coglie da Ortega, in quanto lettore di Spinoza, è però uno spinozismo eterodosso. Ovvero uno spinozismo che è la storia della moltiplicazione e della dispersione dei suoi effetti. Il suo interesse filosofico per Spinoza, è di fatto diverso da quello di Ortega: l'autrice intende studiare soprattutto la salvezza in Spinoza, che a suo avviso è il salvarsi con la separazione l'individuo dal Tutto per lasciare la singolarità come modo di essere, e poi ritirarsi nell'unità assoluta. Salvarsi dallo scorrere (transcurrir) che è la condizione per giungere alla quiete dell'Assoluto. In questo si colloca l'interesse per il Deus sive natura e per l'assoluta identificazione di «Dio, in natura e in vita umana». In Dio, cioè nella struttura matematica fatta di attributi e modi, sta l'insondabile mistero dell'essere: un incontro con il Tutto che è un incontro con se stessi, ovvero è un ri-conoscere sé stessi. Come scrive, più avanti, è la radicalizzazione dell'amore intellettuale eterno, in virtù del quale Dio ama se stesso attraverso l'esistenza dell'uomo che è il tutto, cioè è amore attraverso il nulla: «Tutta l'Etica è lo sforzo di riassumere la Persona nel mondo [...] ma se cè tale ardore [...] è perché fa parte di una separazione. Spinoza non è cieco di fronte a questa separazione, ma l'interpreta come una divergenza da salvare». 
7/M-272 del 1928, intitolato «Tesis sobre Spinoza»², un lavoro preparatorio e iniziale nel quale però l'autrice già anticipa, in forma di appunti, le questioni fondamentali della sua tesi; il secondo è La salvación del individuo en Espino$s a^{3}$ pubblicato nel 1936, un articolo compiuto nel quale l'autrice presenta una riflessione più organica della filosofia spinoziana.

Qui si sviluppano solo alcune delle questioni presenti nel manoscritto ancora inedito ${ }^{4}$.

Il manoscritto è costituito di ventinove pagine. E in esso sono presenti le questioni e le tematiche che sono a fondamento del progetto filosofico zambraniano.

La pagina iniziale, o anche Bozza ha per titolo DISEÑO DE LA VIDA QUE TRANSCRIBE LA ETICA DE ESPINOSA. Essa si articola in tre capitoli:

1. Mundo de Espinosa (suddiviso poi in due paragrafi A. Mundo metafísico; B. Mundo histórico);

2. El sistema de Espinosa desde un mundo problemático. La conversión.

3. Diseño de la vida que la Etica de Espinosa formula. Vida como exstasis en la divinidad.

[2] Nello stesso fascicolo che raccoglie A.F.M.Z 7/M-272, quasi interamente dedicato allo studio della filosofia del filosofo olandese, curiosamente è conservata anche una poesia dal titolo Poesia del cancionero de Amberes. Che Zambrano conservi distrattamente i suoi scritti e li cataloghi, a volte, vagamente, è cosa nota ai suoi interpreti. In questo caso, poi, la poesia conferma la assoluta sua estraneità dalla speculazione che l'autrice presenta su Spinoza. Al di là di ogni considerazione, la poesia merita ugualmente una segnalazione. In questa direzione l'indispensabile saggio dell'autrice danese A. BUNDGÅRD, Un compromiso apasionado. Maria Zambrano: una intelectual al serivicio del pueblo (1928-1929), Madrid, Editorial Trotta, 2009. Si offre una lettura precisa e seria degli eventi politici e storici che determinarono l'avvento prima di De Rivera, della Guerra civile e poi del Franchismo. Puntualmente Bungård sottolinea l'importanza della «circostanza storica» senza la quale non si potrebbe comprendere il pensiero di Zambrano e scrive: «El estudio y comprensión del pensamiento social de Maria Zambrano presupone el conocimiento del panorama político y cultural en el que se hallaban inscritos los intelectuales del periodo inmediatamente anterior a la proclamación de la segunda Republica española», Ibid, p. 19.

[3] Va precisato che il saggio è inserito nel seguente volume, M. Zambrano, Los intelectuales en el drama de España y escritos de la guerra civil, Madrid, Trotta, 1998, pp. 59-69. Il nome del filosofo Espinosa e non Spinoza è utilizzato dalla stessa autrice.

[4] Interessanti sono i due saggi raccolti nel volume di C. Revilla (editora), Claves de la razón poetica, un pensamiento en el orden del tiempo, Madrid, Editorial Trotta, 1998; il primo di L. LLEVADOT, «Zambrano-Spinoza: elementos y transitos del pensar», pp. 139-148; e il secondo di V. M. PINEDA, «Sacrificio, agonia y salvación del individuo (sobre el Spinoza de Maria Zambrano)», pp. 149-158. 
Nel primo capitolo l'autrice segnala l'importanza innanzitutto dell'Etica. A suo avviso, l'opera merita di essere studiata soprattutto perché può essere un modello per una vita filosofica. Di fatti l'etica spinoziana, non solo è distante dalla filosofia cartesiana, ma rifiuta la riduzione dell'uomo ad una mera coscienza che pensa, essendo lontana da una ragione che scivola nel dogma del dubbio.

Per Spinoza la conoscenza è innanzitutto vita, e per questo l'uomo libero non può pensare alla morte, bensì alla vita come anche sostiene Spinoza: «Un hombre libre en nada piensa menos que en la muerte, y su sabiduría no es una meditación de la muerte, sino de la vida $»^{5}$. Per Zambrano, Spinoza non è un autore ingenuo, poiché sa che la conoscenza umana è inadeguata fino a quando non si libera dei suoi appetiti e non riconosce l'origine e la causa delle passioni ${ }^{6}$. L'Etica mostra, da un punto di vista epistemologico e ontologico, un percorso che se adottato permette di uscire dalla fragilità e dalla ignoranza umana.

Per Zambrano, l'etica spinoziana segna un punto di svolta soprattutto rispetto a Cartesio, in un senso preminentemente esistenziale, e capovolge la tradizionale visione cristiana che assegna all'essere umano una superiorità rispetto agli altri enti viventi.

Lo sforzo di Spinoza è quello di porre l'uomo, in quanto un modo tra gli altri modi finiti dell'esistente, all'interno della totalità divina, del Deus sive natura. L'uomo non è sostanza, bensì è un modo come tutti gli altri modi della natura naturata. E quando si dice che l'uomo non è sostanza si dice qualcosa di estremamente grave e scandaloso per la coscienza comune, per il modo stesso con cui oggi si rappresenta l'uomo. Ebbene l'uomo è un modo ma è anche noto che i modi sono determinazioni della sostanza relative all'essenza della sostanza stessa. Allora, da che cosa è costituita l'essenza della sostanza? Per Spinoza l'essenza della sostanza è costituita da tutto ciò che appartiene necessariamente ad un essere assoluto e perfetto. Nulla può mancare ad un essere assoluto e perfetto in quanto assoluto e perfetto. Pertanto alla sostanza appartengono infiniti attributi. Gli attributi sono ciò che costituisce ed esprime l'essenza della sostanza. L'uomo conosce soltanto due attributi della sostanza in quanto partecipa di questi due attributi, ossia il pensiero e l'estensione e assumendo questi due attributi come esemplificazioni è importante dire allora che la sostanza è in quanto sostanza, tutta estesa ed è, simultaneamente, in quanto sostanza tutta pensante la medesima sostanza. Tra pensiero ed estensione in quanto infiniti nel loro genere non c'è nulla in comune ovvero l'uno esprime

[5] B. SPINozA, Ética, IV, LXVIII: «Un hombre libre en nada piensa menos que en la muerte, y su sabiduría no es una meditación de la muerte, sino de la vida».

[6] B. SpINoza, Ética, IV, prefacio: «Los hombres son, sin duda, conscientes de sus acciones $\mathrm{y}$ apetitos, pero inconscientes de las causas que los determinan a apetecer algo». 
tutte le perfezioni del pensiero; l'altro tutte le perfezioni dell'estensione, ma in quanto sono perfezioni della medesima esistenza.

I modi non possono essere che modificazioni della sostanza secondo serie infinite di determinazioni finite in ciascuno attributo o rispetto a ciascuno attributo. Allora, si domanda Zambrano, l'uomo in quanto modo che cos'è?

È come sostenne Cartesio una relazione di corpo e mente? Certamente non relazione di due sostanze intese come corpo e come mente e neppure propriamente relazione di due modi intesi spinozianamente, ossia di un modo dell'estensione che è il corpo e di un modo del pensiero che è un'idea, come ad esempio l'idea di questo corpo. Non è in termini di relazione che bisogna pensare il rapporto mente/corpo. Spinoza dice espressamente che l'uomo è una sola e medesima cosa che può essere pensata simultaneamente sotto l'attributo del pensiero o sotto l'attributo dell'estensione. Dunque non due cose o la relazione o l'intima relazione, come alcuni dicono, di due cose, qui infatti non c'è intimità che tenga. L'uomo è una sola cosa che può essere simultaneamente espressa sotto un attributo o un altro attributo, che può essere simultaneamente considerata sotto un attributo o sotto un altro attributo, ma è evidente che per essere una sola cosa e medesima cosa che simultaneamente può essere considerata sotto un attributo, o un altro attributo in sé, questa cosa non può essere né pensante né corporea perché se fosse pensante non potrebbe essere corporea e se fosse corporea non potrebbe essere simultaneamente pensante.

Dunque cos'è questa unica e medesima cosa che costituisce l'uomo? Nella terza parte dell'Etica Spinoza afferma che l'essenza dell'uomo è espressa da quella che con in latino è la cupiditas, un termine di difficile traduzione e che, in genere, è tradotto con desiderio, ma che è più esattamente la forza con la quale questo ente particolare che è l'uomo tende a conservare se stesso. Si noti che tut to ciò che esiste è mosso dalla forza, dalla tendenza, dal conatus a conservare se stesso. E il conatus che muove tutte le cose a conservare se stesse nell'uomo è la cupiditas e la cupiditas è il primo e fondamentale degli affetti. Zambrano riconosce a Spinoza questo radicale spostamento di posizione dell'uomo da un centro antropocentrico ad uno cosmico-centrico: «toda la Ética es el esfuerzo por reasumir la persona en el mundo ${ }^{7}$. Come si comprende, la filosofia di Spinoza si presenta propriamente come una filosofia anti-umanistica, se per umanesimo si intende quella posizione del pensiero che considera l'uomo in quanto fine della creazione, centro dell'universo, e anello di congiunzione del materiale e dello spirituale e si considera l'uomo come una sorta di stato nello stato, come una sorta di eccezione alle leggi della natura. L'uomo come tutte

[7] M. Zambrano, «La salvacion del individuo en Espinosa», in Los intelectuales en el drama de España y escritos de la guerra civil, Madrid, Trotta, 1998, p. 69. 
le altre cose obbedisce alle leggi della natura e deriva necessariamente da Dio per le stesse leggi e con la stessa necessità per cui derivano da Dio tutte le cose.

Per giungere ad una prima conclusione, Zambrano seguendo Spinoza chiosa in questo modo: «tutto ciò che esiste, in quanto esiste così come esiste, è perfetto».

Ma come può l'Etica diventare una Guida per l'ente? Secondo Zambrano, la conoscenza spinoziana nell'Etica, mostra il metodo per un'etica della conoscenza; essa è una guida, innanzitutto, perché l'Etica è un "sapere dell'anima», un saber sobre el alma, distante dal quel sapere logico-razionale cartesiano ${ }^{8}$, e assolutamente prossimo, invece, al sapere esistenziale che già Ortega appellava «spontaneità» e che è la più nobile manifestazione del pensiero spagnolo 9 .

\section{Lo Esquema.}

Passando ora all'altro punto cruciale del manoscritto dove la lettura su Spinoza si intreccia a Hegel, già nello Esquema che segue la Bozza sono evidenziati tre punti fondamentali:

1.Tratado de las Pasiones (Spinoza);

2. Contrareforma: Ejercicios espirituales de Espinosa.

3. E una conclusione dal titolo El Tiempo.

Ciò che ci sembra interessante è proprio quest'ultima questione: quella sul tempo.

Infatti, una riflessione sul Tempo risulta importante soprattutto in Spinoza il quale se ne serve per criticare la filosofia cartesiana che, a suo avviso, non era riuscita a spiegare adeguatamente l'origine del tempo.

Nel suo Cogitata metaphysica (opera incompiuta del 1663) Spinoza dichiara: «que Dios es «causa libre» pues siendo causa natural, no se explicaría el tiempo» ${ }^{10}$. Idea che poi riprende nell'Etica dove unendo il concetto di «causa

[8] Nel suo scritto La confesion: genero literario Zambrano scrive: «La filosofía moderna, la que nace con Descartes, ha abandonado la exigencia de que la vida se convierta [...] Solo un filosofo alejado, Spinoza, dedica la atención merecida a la reducción de la vida en el libro IV, sobre las pasiones [...]. El drama de la cultura moderna ha sido la falta inicial de contacto entre la verdad de la razón y la vida. Porque toda vida es ante todo dispersión y confusión, y ante la verdad pura se siente humiliada. Y toda verdad pura, racional y universal tiene que encantar a la vida; tiene que enamorarla", $C f$. M. Zambrano, La confesion: genero literario, Madrid, Mondadori, 1988, p. 8.

[9] Cf. J. Ortega y Gasset, Lorigine sportiva dello Stato, Milano, Se, 2007, p. 12.

[10] Dal foglio dello Esquema del M :«Despues en la Etica identifica «causa libre» con «Causa natural». Entonces ¿cómo se explica el tiempo? (pag. 79 de Couchoud)». 
libre» con quello di «Causa natural» conclude dicendo che Dio è causa di tutta la Natura e per questo anche del Tempo.

Ma perché Zambrano è così interessata al tempo? Si potrebbe dire che la riflessione sul tempo segna un momento significativo della sua filosofia. E nello specifico, segna il momento di massimo allontanamento dal suo maestro, e avvicinamento a Xavier Zubiri. Ovvero, a quel professore che nel 1934, trasferito in Germania, lascia la cattedra di Metafisica alla giovane filosofa ${ }^{11}$.

In questo orizzonte, Zubiri, in particolare con la sua razón-sentiente, segnala una riflessione importante sul Tempo. Egli qualifica il tempo innanzitutto come la struttura fondamentale della realtà umana. La durata, la permanenza e la durata del fluire e dello sforzo sono per il filosofo i distinti modi del tempo che rimandano al concetto di vita. Zambrano parte da questa tesi e la rielabora prima nel saggio "La multeplicidad de los tiempos», nei capitoli «La persona humana» e poi in particolare in «El tiempo» di Persona y Democracia dove "circoscrive» il Tempo in una curiosa definizione: "El tiempo que es desgarramiento del ser» ${ }^{12}$. Il tempo va pensato in relazione ad uno strappo in relazione alla rottura che si compie nell'essere, solo in questo modo il tempo è e diviene.

La questione che ora Zambrano solleva è delicata. L'autrice attribuisce a Dio, l'essere, la sostanza assoluta e unico essere che è causa di sé , la nascita del Tempo.

Questa anticipazione filosofica verrà ripresa meglio diversi anni dopo quando scrivendo in una delle sue opere più fortunate, L'Uomo e il Divino, un'elaborazione sul tempo l'autrice si servirà della filosofia di Hegel per affermare quanto già la filosofia di Spinoza le aveva consegnato.

In questo scia più hegeliana che spinoziana, il tempo in quanto anche coscienza storica, per Zambrano: «[...] es la característica del hombre de nuestros días. El hombre ha sido siempre un ser histórico» ${ }^{13}$. uesta affermazione, già presente negli Apuntes del manoscritto del 1931-32 ${ }^{14}$, qui diventa il presupposto

[11] G. Gómez Cambres, «Zubiri, amigo y maestro de María Zambrano», in Actas III Congreso Internacional sobre la vida y obra de María Zambrano: María Zambrano y la «Edad de Plata» de la cultura española, Fundacion María Zambrano, Vélez-Málaga 2004, pp. 173-180.

[12] M. Zambrano, «Persona y democracia» in Obras completas III (1955-1973). El hombre y lo divino; Persona y democracia, La España de Galdos, España de Galdos, España, sueño y verdad, Los sueños y el tiempo, El sueño creador, La tumba de Antigona, edicion dirigida por J. Moreno Sanz, Barcelona, Galaxia Gutemberg, 2011.

[13] Come poi Zambrano precisa nel primo capitolo «Perplejidad ante la historia. La conciencia histórica. El tiempo» del suo lavoro Persona y democracia: «El tener lo que se ha nombrado»conciencia histórica» es la característica del hombre de nuestros días. El hombre ha sido siempre un ser histórico», in Obras Completas III, p. 381.

[14] E nelle quattro pagine, conservate all'interno di questo Manoscritto, segnato solo da una data II novembre, Zambrano appunta:» En Espinosa combaten dos sentidos de razón. La razón 


\section{etico-politico della storia ${ }^{15}$ che a suo avviso, in questa continua gestazione, si}

naturalista de la divinidad identificada con la naturaleza, razón da causa a efecto, razón matemática aplicada a la naturaleza y la razón que más tarde había de declararse en el idealismo, razón del hombre, no razón natural, que vemos apuntar en su teoría del conocimiento en el Libro II de la Ética. El que en la Ética espinosiana se afirme ante todo la unidad y la unicidad de la substancia, el que se eche mano de ella continuamente para probar las proposiciones que se presentan a lo largo del libro, ha hecho que se interprete el pensamiento de Espinosa desde el punto de vista de la substancia, como si a él le hubiera interesado exclusivamente afirmar la existencia de ella, como si hubiera partido su busca. Lo que sucede es más bien lo contrario: la substancia, por lo mismo que es todo, no basta para explicar todos los problemas que la Ética de Espinosa contiene y dentro de ellos el del conocimiento. La afirmación de la substancia tiene el sentido de afirmar antes que nada que algo es y que únicamente dentro este algo que es, tiene sentido el ser de las demás cosas, es un encajar el ser del hombre en este otro ser de que se parte afirmándole de modo total y absoluto. (p. 1). Pero una vez afirmado esto, comienza justamente el problema, pues, ¿Qué ser, que realidad a tener los demás seres dentro de esa única substancia? El concepto de substancia en Espinosa es punto de partida, pero no de llegada. Los problemas comienzan justamente después de esa evidencia, de esa verdad indubitable, casi dogma que Espinosa afirma al comienzo de su Ética: la existencia de algo que es en si y que en si se concibe. Pero el problema de Espinosa no es la substancia, el problema es el de encajar y ver qué clase de relación hay entre el ser único y el ser del hombre, cuyo res no es independiente. El ser del hombre no puede tener más realidad que la que le permita dentro de si la estructura de esta única realidad de la substancia, dentro de la cual tiene que estar alojado metafísicamente. El problema está en el conflicto entre «el ser que es por sí» y el hombre que no es por sí, que no es necesario en su existencia. Relación que nos hace pensar en la relación entre la omnipotencia de Jeova y la inanidad del hombre que ante él no es nada. El problema de la salvación individual, que no se concibe sin el de la libertad es el de Espinosa. ¿Existe la libertad? Tiene el hombre un ser que la permita? (p. 2) Es el hombre necesario? Cómo se articula este ser del hombre con el absoluto ser de la substancia? Todo gira en torno a esta decisiva cuestión: hacer necesaria la vida del hombre «cuya esencia no envuelve su existencia». Ir en busca de esta necesidad o seguridad para la fluctuante vida humana, necesidad que es justificación, justificación que es seguridad, sosiego, vida feliz en suma, es lo que busca Espinosa. En la idea de substancia como punto de partida encontramos un valor dogmatico, funciona a manera de dogma, aunque su relación con la mente que lo piensa no sea la misma que en el dogma. Pues la evidencia de esta verdad primera no es evidencia de la fe, sino evidencia de la razón humana, que encontraría dentro de sí misma esta verdad que es la misma de Dios de la Biblia. Por eso vemos en Espinosa antes que nada un intento de fundar la religión en la razón, de dar a la fe un supuesto de evidencia racional. "Yo soy el que ES» dijo el Dios de Abraham y de Jacob, que ahora existe pensado como substancia. Es la absoluta omnipotencia divina, el «misterium tremendum» pensado bajo la suprema categoría del ser tradicional, de la substancia aristotélico-escolastica. (p. 3). Dentro de ella va a tener que explicarse todo lo existente y para ello habrá que echar mano de la estructura interna de la substancia. Dentro de este único ser se diversifican las diversas capas y zonas del ser que van a permitir la existencia de otro seres, cuya existencia es necesario explicar y justificar (p. 4).

[15] Come Zambrano scriverà in El hombre y lo divino, Madrid, Siruela, 1992, p. 13: «Hace muy poco tiempo que el hombre cuenta su historia, examina su presente y 
orienta verso un futuro e in vista della salvezza dell'umanità ${ }^{16}$, appunto della salvación del individuo.

Concludendo, Zambrano giunge, dunque, alla formulazione del Tempo grazie a Zubiri e sempre dopo aver letto con Ortega, sia Spinoza che Hegel. Sebbene in tutte le sue opere Zambrano non nominerà mai volentieri il filosofo di Stuttgart, se non nel saggio El hombre y lo divino, già nell'introduzione si domanderà: "Cos'è lo storico? Cos'è ciò che attraverso la storia si fa e si disfa, si desta e si assopisce, appare per sparire? È sempre altro o sempre lo stesso al di sotto di ogni avvenimento? E stato Hegel a formulare, prima della domanda, la risposta. In quanto scoprì la storia come vicissitudine necessaria, inesorabile, dello spirito» ${ }^{17}$.

Paola Coppi is Ph.D. in Philosophy at the University of Verona.

\section{RESEARCH INTERESTS: \\ Areas of Specialization: Schelling and Spinoza, Ortega y Gasset and María Zambrano.}

proyecta su futuro sin contar con los dioses, con Dios, con alguna forma de manifestación de lo divino», tr. it. M. Zambrano, L'uomo e il divino, Roma, Ed. Lavoro, 2009, p. 9.

[16] Manoscritto conservato nella Fundacion María Zambrano di Vélez-Málaga sotto la catalogazione: M-01 AFMZ 102 cuartillas Apuntes de historia de la Filosofía de Xavier Zubiri. Esso raccoglie diversi Apuntes. Nel n. 9, Mecanografiado Politica, Zambrano scrive: «Toda política supone una conciencia histórica, es la gestación de la historia, su alumbramiento. Toda política se dirige a un futuro, lo crea. Y como actividad humana es posible y no necesaria, porque en lo humano existe la gloria y la tragedia de la posibilidad, de la libertad, de la indeterminación. De no ser asi lo humano, no habría política que supone ya en su base, libertad de caminos, posibilidad de diversos fines. Tampoco habría historia.La historia no es sino un dialogo bastante dramático por cierto entre el hombre y lo que no es el. Gracias al hombre hay dialogo, dualidad, el es siempre lo otro en la naturaleza. Ella permanece fiel a su impulso creador, en todo sus acontecimientos hay un carácter de necesidad y en su silencioso ser es la máxima virtud la obediencia, la entrega sumisa a los latentes designios, el hombre no. Emerge de la naturaleza, habla, contraria el orden hallado...». E qui termina la citazione priva anche di una numerazione delle pagine. $\grave{E}$ interessante notare, dunque, non solo l'influsso di Spinoza su Zambrano, ma anche il riferimento qui più mirato per la natura e all'uomo in Hegel. Per un maggior approfondimento, si rimanda alla lettura puntuale e attenta del prezioso saggio di JUAN J. PADIAL, «Los hábitos y la emergencia de la subjetividad. La autoconciencia y la cultura según Hegel», in Annuario Filosofico XXIX, Milano, Mursia, 2013, pp. 139-151. In tal senso, unaltra lettura imprescindibile della filosofia hegeliana è offerta dal prezioso volume di R. Finelli, Mito e critica delle forme. La giovinezza di Hegel 1770-1801, Roma, Editori Riuniti, 1996.

[17] M. Zambrano, L’uomo e il divino, p. 10, op. cit. 
Areas of Competence: History of Theology and Philosophy in the Twentieth Century, the Philosophy of Religion, the Spanish and German philosophy, Politics of Philosophy and Gender.

\author{
ADDREss: \\ Via San Salvatore Corte Regia 4 \\ 37121 Verona \\ Italia.
}

\title{
Recent Publications:
}

- «María Zambrano: la democracia humanizada», in H. BiAgini y D. A. Fernández Peychaux, Democracia, Neoliberalismo y Pensamiento politico alternativo. Actas del v Coloquio Internacional de Filosofía Política. 2013, Editorial de la Universidad Nacional de Lanús, 2015, pp. 240-242.

- «María Zambrano, filósofa del sur, pensadora del exilio». In Jornadas Internacionales de Hispanismo Filosófico (11. 2013, Universidad de Granada), Filosofías del Sur, Madrid, Fundación Ignacio Larramendi, 2015.

- «María Zambrano. La Democracia humanizada», in A. CAmpillo y D. ManZANERo, Los retos de la Filosofía en el siglo XXI. Actas del I Congreso internacional de la Red española de Filosofía, València, Publicacions de la Universitat de València (PUV), 2015, pp. 63-68.

-«Riflessioni «libere» a partire dal femminile in Adriana Cavarero», in: R. PIsCONTI (a cura di) Riflessioni critiche sul femminismo contemporaneo, Villasanta (Mb), Limina Mentis, 2014, pp. 223-257.

- «Un’estranea e altra prossimità'. Riflessioni a partire dalla filosofia di Maria Zambrano». In AA.VV., Differenze e relazioni. Il prossimo e l'estraneo, Aracne Editrice, Roma, 2014, pp. 177-182. 